\title{
INVARIANTS OF THE EULER EQUATIONS FOR IDEAL OR BAROTROPIC HYDRODYNAMICS AND SUPERCONDUCTIVITY IN $D$ DIMENSIONS
}

\author{
B.A. KHESIN and Yu.V. CHEKANOV \\ Differential Equation Division, Department of Mathematics, Moscow State University, Moscow 119899, USSR
}

Received 15 December 1988

Revised manuscript received 14 March 1989

Accepted 21 March 1989

Communicated by V.I. Arnol'd

\begin{abstract}
The Hamiltonian formalism for the Euler equations of an ideal fluid, superconductivity and a barotropic fluid on a $D$-dimensional Riemannian manifold is proposed. We show that each of these equations has an infinite series of integrals if $D$ is even ("generalized enstrophies") and at least one integral if $D$ is odd ("generalized helicity"). We prove that the magnetic hydrodynamics integral $\int(\boldsymbol{v}, \boldsymbol{B}) \mu$ is equal to the average linking number of vector fields rot $\boldsymbol{v}$ and $\boldsymbol{B}$ in terms of the ergodic theory. All the invariants considered are Casimir elements (i.e. invariants of coadjoint action) of the corresponding infinite-dimensional Lie algebras.
\end{abstract}

\section{Introduction}

The equations of an inviscid incompressible fluid are Hamiltonian ones on the orbits of the group of volume-preserving diffeomorphisms $[1,2,4]$. It is well known that for a two-dimensional flow there are an infinite number of area integrals $\left(\int f(\operatorname{rot} v) \mathrm{d}^{2} x\right)$ and for a three-dimensional one there is a total helicity integral $\left(\int(\operatorname{rot} v, v) \mathrm{d}^{3} x\right)$.

The primary purpose of this paper is to show that the equations of the ideal fluid have an infinite number of integrals on an arbitrary evendimensional manifold $M(D=\operatorname{dim} M=2 m)$ and (at least) one integral for odd $D$.

The statement mentioned was settled for the standard $\mathbb{R}^{D}$ with the help of some explicit coordinate calculations by Serre (see ref. [15]). His method is based on the Hamiltonian structure of the Euler equations proposed by Olver [13]. We show that Olver's formulation coincides with the one given by Arnol'd (see refs. [2, 4, 7]). Thus we answer the question raised in ref. [13] about the relation of these two approaches. Note that our generalizations differ greatly from those proposed by Dezin in ref. [6] (where the odd-dimensional integral is obtained).

Sections 3 and 4 contain the multidimensional generalizations of superconductivity and barotropic fluid equations. We show that these equations are Hamiltonian ones and find their conservation laws analogous to the hydrodynamical invariants. In the superconductivity case the phase space is identified with the dual space (whose origin is displaced) to the infinite-dimensional Lie algebra of all divergence-free vector fields.

The Euler form of these equations differs from the standard hydrodynamics equation by the Coriolis-type term. Analogous results for the threedimensional superconductivity equations were obtained by Holm and Kupershmidt (see ref. [11]). 
The configuration space of the barotropic fluid is a semidirect product of the diffeomorphisms' group and the space of all functions on the manifold considered. The similarity of the barotropic fluid to the ideal one can be explained by its "incompressibility" in the coordinates connected with the density. Some results on two-dimensional barotropic flows can be found in an article by Holm et al. [12].

In the final section we describe the ergodic interpretation of the three-dimensional magnetic hydrodynamics integral in the spirit of ref. [5]. We show that the invariant $\int(\boldsymbol{B}, \boldsymbol{v}) \mathrm{d}^{3} x$ coincides with the average linking number of the rotor vector field rot $\boldsymbol{v}$ and the magnetic vector field $\boldsymbol{B}$. One of the crucial points of ref. [5] is the ergodic interpretation of the total helicity. The ergodic interpretation of multidimensional (magneto-) hydrodynamics integrals is an open and intriguing problem.

All the integrals in question are invariants of the coadjoint representation of the corresponding Lie groups (the so-called Casimir elements), i.e. they do not depend on the particular choice of the Hamiltonian. This opens the way to the investigation of the nonlinear stability problems by Routh's method [12]. We also think that information about the orbits can be of help in the study of the Cauchy problem of multidimensional hydrodynamics.

Note that the existence of an infinite number of integrals for the flow of an even-dimensional fluid does not imply a complete integrability of the corresponding hydrodynamics equations. The invariants considered only define the coadjoint orbits (generally speaking, infinite-dimensional), on which the evolution takes place. For the equations on this manifold-orbit there is a unique energy integral while the integrability requires an infinite number of them.

Information about the orbits of the coadjoint action (and, hence, about the geometry) of the corresponding infinite-dimensional Lie groups is probably more important in itself than its hydrodynamics applications.

\section{Hydrodynamies on the Riemannian manifold}

Let $M^{D}$ denote a compact Riemannian manifold (without boundary) and $\mu$ its volume form (which, in general, has no connection with the form induced by the metrics $(.,$.$) ). The equation$ of the incompressible fluid on $M$ is:

$$
\begin{aligned}
& \dot{v}=-(v, \nabla) v+\nabla p \\
& \operatorname{div} v=0,
\end{aligned}
$$

where $v$ and $p$ are a time-dependent vector field and a function on $M,(v, \nabla) v$ denotes the covariant derivative $\nabla_{v} v$ for Riemannian connection.

Theorem 1. Eq. (1) has

(i) the integral

$I(v)=\int_{M} u \wedge(\mathrm{d} u)^{m}$

in the case of an arbitrary odd-dimensional manifold $M(D=2 m+1)$;

(ii) an infinite number of integrals

$I_{f}(v)=\int_{M} f\left(\frac{(\mathrm{d} u)^{m}}{\mu}\right) \mu$

in the case of an arbitrary even-dimensional manifold $M(D=2 m)$, where $u$ is a 1 -form induced from $v$ by the "lifting of indices" defined by the metrics

$u(\xi)=(v, \xi) \quad \forall \xi \in T_{x} M$,

and $f$ is an arbitrary function of one variable.

Proof. Let $\mathrm{G}$ be the group of all diffeomorphisms preserving volume $\mu$ and $\mathscr{G}$ be the Lie algebra of all divergence-free vector fields. Let $\Omega_{k}$ and d $\Omega_{k-1}$ denote the spaces of all $k$-forms and exact $k$-forms on $M$, respectively.

Lemma 1. (See also ref. [7].) There exists a natural isomorphism between spaces $\mathscr{G}^{*}$ and $\Omega_{1} / \mathrm{d} \Omega_{0}$. The 
corresponding pairing is

$\langle[u], v\rangle=\int_{M} u(v) \mu$

where $v \in \mathscr{G}$, and the form $u \in \Omega_{1}$ is an arbitrary representative of $[u] \in \Omega_{1} / \mathrm{d} \Omega_{0}$.

Proof. The exact forms correspond to the zero functional, since

$\int_{M} \mathrm{~d} f(v) \mu=\int_{M} \mathrm{~d} f \wedge i_{v} \mu=0$

The vanishing of this integral follows from the fact that the G-invariance of $\mu$ implies the closeness of $i_{v} \mu$ and, hence, the exactness of $\mathrm{d} f \wedge i_{v} \mu$. Thus, the definition of the change of variables in the integral and the invariance of $\mu$ imply the coincidence of the coadjoint G-action with the $\mathrm{G}$-action on the space of 1 -forms.

Proposition 1. The following integrals on $\mathscr{G}^{*}$ are invariants of the coadjoint action:

(i) in case $D=2 m+1$

$I([u])=\int_{M} u \wedge(\mathrm{d} u)^{m}$,

(ii) in case $D=2 m$

$I_{f}([u])=\int_{M} f\left(\frac{(\mathrm{d} u)^{m}}{\mu}\right) \mu$

where $f$ is an arbitrary function of one variable.

Remark 1. Obviously, the given functionals are properly defined on $\mathscr{G}^{*}$, i.e. they do not depend on the choice of the representative in class [u].

Proof. Since the coadjoint action coincides with the change of variables (see lemma 1), our statement follows from the coordinate-free definition of the corresponding integrals.
Definition 1. The standard linear Poisson structure on $\mathscr{G}^{*}$ maps each functional $F$ on $\mathscr{G}^{*}$ to its Hamiltonian equation (called Euler-Arnol'd's equation, see refs. $[1,4]$ ),

$[\dot{u}]=-\operatorname{ad}_{\delta F / \delta[u]}^{*}[u]$,

where the variational derivative $\delta F / \delta[u] \in \mathscr{G}$ is defined by the relation

$\left.\frac{\mathrm{d}}{\mathrm{d} \varepsilon} F([u]+\varepsilon w)\right|_{\varepsilon=0}=\langle\delta F / \delta[u], w\rangle$

for each $w \in \mathscr{G}^{*}$.

Note that operator $\mathrm{ad}_{v}^{*}[u]$ coincides (by proposition 1) with the operator $L_{v}[u]$ of the Lie derivative determined by the vector field $v$ on $M$ (the definition is correct since $L$ commutes with d).

Definition 2. Let (.,.) be the Riemannian metrics on $M$ (whose volume form in general differs from the given volume $\mu$ ). It defines a nondegenerate scalar product on $\mathscr{G}$ :

$(v, w)_{\mathscr{S}}=\int(v, w) \mu$

and, hence, an invertible operator $A: \mathscr{G} \rightarrow \mathscr{G}^{*}$, called the inertia operator (see ref. [4]). It maps the vector field $v$ to class $[u$ ], satisfying the following relation:

$(v, .)_{\mathscr{G}}=\int_{M} u(.) \mu$

where $u$ is an arbitrary representative of $[u]$. Thus, $\mathscr{G}^{*}$ inherits from $\mathscr{G}$ the nondegenerate scalar product $(., .)_{\mathscr{G} *}$. We define on $\mathscr{G}^{*}$ the Hamiltonian function

$H([u])=\frac{1}{2}([u],[u])_{\mathscr{G *}}$

Since $H$ is quadratic, its variational derivative is $\delta H / \delta[u]=A^{-1}([u])$. Hence, the Hamiltonian equation for $H$ coincides with $[\dot{u}]=-L_{v}[u]$, where $[u]=A v$. 
In this equation we may pass from the classes to the particular representatives: $\dot{u}=-L_{v} u+\mathrm{d} \psi$. Operator $A^{-1}$ transforms it into the following equation on $\mathscr{G}$ :

$\dot{v}=-(v, \nabla) v+\mathrm{d} p$

called the Euler equation of the ideal incompressible fluid (see refs. [1, 4]).

The equation $\dot{\omega}=-L_{v} \omega$ on the space of all exact 2-forms $(\omega=\mathrm{d} u)$ is called the Helmholtz curl equation. The same equation with a more coordinate-like definition appears as Olver's Hamiltonian formulation (see eq. (3.9) of ref. [13]).

Lemma 2. Invariants $I([u])$ and $I_{f}([u])$ for $[u]=$ $A v$ are constant on the trajectories of the Euler equation.

Proof. The Hamiltonian character of $[u]$ implies its tangency to the orbits of the coadjoint Gaction on $\mathscr{G}^{*}$. Hence, $I_{f}([u])$ and $I([u])$ are its integrals. Lemma 2 and, hence, theorem 1 are proved.

For example, in the standard metrics of $\mathbb{R}^{3}$, integral (2a) coincides with

$I(v)=\int(v, \operatorname{rot} v) \mu$

and for $\mathbb{R}^{2}$ integral (2b) coincides with

$I_{f}(v)=\int(\operatorname{rot} v) \mu=\int f\left(\Delta h_{v}\right) \mu$,

where $h_{v}$ is the "flow function" of the vector field $v$ relative to the symplectic form $\mu$ (see ref. [4]).

Remark 2. The invariant (2b) of the plane-parallel $2 m$-dimensional flow induced by $(2 m-1)$ dimensional flow is trivial, since $(\mathrm{d} u)^{m}=0$. Therefore, the reduction of dimension gives no integrals different from $(2 a)$.
Remark 3. The integrals $I([u])$ and $I_{f}([u])$ do not form a complete set of continuous invariants of coadjoint orbits. By analogy with two- and threedimensional cases [5], it is possible to construct parametrized families of orbits with equal values of these functionals.

Remark 4. The manifold $M$ may be multiconnected. In the nonsimply connected case the cohomological class of $[u]$ is also an invariant (compare ref. [3]). Other examples of discrete invariants of the Euler equation are the number of points on $M$ where $\mathrm{d} u$ degenerates and the orders of its degeneracy (here $[u]=A v)$.

Remark 5. The manifold $M$ may be noncompact or may have a boundary (we may consider $M=$ $\mathbb{R}^{D}$ ). In general, we should consider vector fields tangent to the boundary.

The rest of the section is devoted to the case of the odd-dimensional fluid. Theorem 1 provides in this case the existence of one invariant. The geometrical approach to its proof allows to obtain the following statements.

Corollary 1. On an odd-dimensional manifold ( $D$ $=2 m+1$ ) the curl vector field (i.e. the kernel of $\left.(\mathrm{d} u)^{m}\right)$ is "frozen in the fluid."

Proof. The rotor vector field $\omega$ is defined by the condition $i_{\omega} \mu=(\mathrm{d} u)^{m}$.

Class $[u]$ (and, hence, $\left.(\mathrm{d} u)^{m}\right)$ is transported by the flow; volume $\mu$ is invariant and, hence, the vector field $\omega$ is also transported geometrically (i.e. is "frozen in the fluid").

Corollary 2. (For $D=3$ see ref. [5].) The equation of an odd-dimensional incompressible fluid has a set of integrals:

$I_{\mathrm{c}}(v)=\int_{\mathrm{c}} u \wedge(\mathrm{d} u)^{m}$ 
where the integral is taken over each ergodic component of the momentary curl vector field.

The proof follows immediately from the Stokes formula and from the observation that the restriction of $(\mathrm{d} u)^{m}$ on the boundary of any ergodic component vanishes.

Corollary 3. Let $v$ denote a vector field of a flow of an incompressible fluid on an odd-dimensional manifold and $f$ a function preserving its values for each fluid particle, i.e. $L_{v} f \equiv 0$ (for example, $f$ is one of the Lagrangian coordinates of the fluid). Then the flow "in the general position" is "completely integrable" in the following sense. There exist $D$ functions independent of $M$ almost everywhere, forming a complete set of Lagrangian coordinates. In other words, knowing the solution of the Euler equation (the time dependence of the velocity) and knowing one of the Lagrangian coordinates, we can calculate the other ones. So, we can define the location of any fluid particle at an arbitrary moment avoiding integrating the velocity.

Proof. Since the curl vector field $\omega$ and function $f$ are "frozen in the fluid" for the flow corresponding to $v$, the derivative of $f$ along $\omega$ is "frozen" too, i.e. it is transported as a function. Iterating the procedure, we obtain for "general" $f$ and $\omega$ the functions

$$
f, L_{\omega} f, L_{\omega} L_{\omega} f, \ldots, \underbrace{L_{\omega} \ldots L_{\omega}}_{2 m},
$$

which are almost everywhere functionally independent and thus can be used as Lagrangian coordinates.

Remark 6. A three-dimensional manifold $M$ is decomposed into a finite number of cells by the critical levels of some function $f$ corresponding to a stationary flow $v[1,5]$. In any of these cells diffeomorphic to the product of a torus and of an interval, fields $v$ and $\omega=\operatorname{rot} v$ are tangential to the level surfaces of $f$ (which are diffeomorphic to a torus) and define there periodic and quasi- periodic motions. Choosing $f$ as one of the Lagrangian coordinates, we obtain $L_{\omega} f \equiv 0$ (by the tangency condition of the vector field $\omega$ and the level surfaces of $f$ ), i.e. the second Lagrangian coordinate constructed by this method is trivial. Hence, an ergodic behavior of the flow on some of the tori is possible.

Remark 7. In the case of $D=3$ the integral $I(v)$ has an ergodic interpretation as the average selflinking number of rot $v$ (see ref. [5]). For $D>3$ a similar interpretation is unknown.

\section{The generalized superconductivity equation}

The connection of superconductivity to the equations of the incompressible fluid is due to the fact that at a high density an electronic gas is similar to a fluid. Indeed, the repelling of the particles having equal charges in the electronical clusters makes the gas incompressible.

The equation of (nonrelativitic) superconductivity in $\mathbb{R}^{3}$ is

$$
\dot{v}=-(v, \nabla) v-v \times B+\nabla p,
$$

where $v$ denotes a divergence-free field of the electronic gas velocity, $\boldsymbol{B}$ a constant external divergence-free magnetic field, and symbol $\times$ the vector product for the standard metrics [9].

We define the analog of this equation on an arbitrary manifold. Let $M$ be an arbitrary $D$ dimensional manifold with volume $\mu$ and the Riemannian metrics $g$. We suppose that $v$ is divergence-free with respect to $\mu$, and $B$ is a "strictly divergence-free" $(D-2)$-vector field with respect to volume $\sqrt{g}$, i.e. the substitution of $\boldsymbol{B}$ in $\sqrt{g}$ is exact: $L_{B} \sqrt{g}=\mathrm{d} \alpha$ (for example, in the case of $H_{2}(M)=0$, the condition $\mathrm{d} i_{B} \sqrt{g}=0$ is sufficient). We define "the vector product" of field $v$ and $(D-2)$-vector field $B$ in the standard way:

$v \times B=*(v \wedge B)$, 
where $*$ is the isomorphism of $k$ - and $(D-k)$ vector fields induced by the metrics [8]. We call (3) the generalized superconductivity equation.

Theorem 2. The multidimensional superconductivity equation (3) has

(i) an integral

$I(v)=\int_{M}(u+\alpha) \wedge[\mathrm{d}(u+\alpha)]^{m}$

in the case $D=2 m+1$, and

(ii) an infinite number of integrals

$I_{f}(v)=\int_{M} f\left(\frac{[\mathrm{d}(u+\alpha)]^{m}}{\mu}\right) \mu$

in the case $D=2 m$, where $\alpha$ is a 1-form satisfying the relation $\mathrm{d} \alpha=i_{B} \sqrt{g}$. The 1 -form $u$ is obtained by the "lifting of indices" of field $v$ with the use of metrics $g$.

Lemma 3. The inertia operator $A$ transforms eq. (3) into the following equation on $\mathscr{G}^{*}$ :

$[u]^{*}=-L_{v}[u+\alpha]$,

where $\mathrm{d} \alpha=i_{B} \sqrt{g}, v=A^{-1}[u]$.

Proof. The inertia operator $A$ (see section 1) is the composition of the "lowering-of-indices" operator and of the projection $\Omega_{1} \rightarrow \Omega_{1} / \mathrm{d} \Omega_{0}$. Hence,

$$
\begin{aligned}
A(v \times B) & =A(*(v \wedge B)) \\
& =\left[i_{v \wedge B} \sqrt{g}\right]=\left[i_{v} i_{B} \sqrt{g}\right] \\
& =\left[i_{v} \mathrm{~d} \alpha\right]=\left[L_{v} \alpha\right]=L_{v}[\alpha]
\end{aligned}
$$

(the second identity follows directly from the definition of * (see ref. [8]) and the fifth one from the homothopy formula:

$L_{v}=i_{v} \mathrm{~d}+\mathrm{d} i_{v}$.

Therefore, in analogy with section 2 we obtain eq.
(4) on the quotient space $\Omega_{1} / d \Omega_{0}$. For the representatives of the conjugate classes eq. (4) reads:

$\dot{u}=-L_{\mathrm{v}}(u+\alpha)+\mathrm{d} \psi$.

Lemma 3 is proved.

Proof of theorem 2. Eq. (4) can be written as

$[u+\alpha]^{\cdot}=-L_{v}[u+\alpha]$,

since the constancy of the magnetic field $B$ implies $[\alpha]^{*}=0$. This form of eq. (4) enables us to conclude that $[u+\alpha]$ moves along an orbit of the coadjoint G-action.

The functionals (2a) and (2b) evaluated at $[u+\alpha]$ are constant along the orbits of these points in $\mathscr{G}^{*}$, since the G-action is a change of the variables in the expression $[u+\alpha]$. These orbits coincide with the affine shifts by $[\alpha]$ of the standard orbits of the coadjoint action on $\mathscr{G}^{*}$. In particular, these functionals, evaluated at $[u+\alpha]$, where $[u]=A v$, are invariant along the solution of eq. (3).

Corollary 4. Let $\left[u^{\prime}\right]$ be the new coordinate on $\mathscr{G}^{*}$ different from the standard one by the shift of the origin to the point $[-\alpha]:\left[u^{\prime}\right]=[u+\alpha]$. Then eq. (4) is a Hamiltonian one on coadjoint orbits with the Hamilton function $H=\frac{1}{2}\left(\left[u^{\prime}-\alpha\right],\left[u^{\prime}-\alpha\right]\right)$ with respect to the standard linear bracket on a new $\mathscr{G}^{*}$.

Indeed, eq. (4) is $\left[u^{\prime}\right]^{*}=L_{v}\left[u^{\prime}\right]$ for the new coordinate $\left[u^{\prime}\right]$ and $A v=\left[u^{\prime}-\alpha\right]$.

\section{The multidimensional barotropic fluid}

A barotropic fluid (whose pressure depends only on the density) on a manifold $M$ with metrics $g$ is described by the following system of equations on the velocity $v$ and the density $\rho$ :

$\dot{v}=-(v, \nabla) v+\nabla h(\rho)$,

$\dot{\rho}+\operatorname{div}(\rho v)=0$. 
We use the $D$-form $\theta$ of density $\theta=\rho \mu \in$ $\Omega^{D}(M)$, where $\rho$ is the density function and $\mu$ the standard volume form induced by the metrics $\mu$ $=\sqrt{g}$.

Theorem 3. Eq. (5) of the barotropic fluid admits (i) an integral

$I(v)=\int_{M} u \wedge(\mathrm{d} u)^{m}$

on an arbitrary odd-dimensional manifold $(D=$ $2 m+1)$,

(ii) an infinite number of integrals

$I_{f}(v)=\int_{M} f\left(\frac{(\mathrm{d} u)^{m}}{\theta}\right) \theta$

on an arbitrary even-dimensional manifold $(D=$ $2 m$ ), where $\theta=\rho \mu$ and $u$ is the 1-form obtained from $v$ by the "lifting of indices."

Proof. A heuristic proof of the theorem is based on the fact that the fluid is incompressible with respect to the new volume $\theta$ (depending on time and on the initial conditions), since the density is transported by the flow. Thus, we can apply theorem 1, whose assumptions demand no connection between metrics and volume. The rest of the section contains a more detailed consideration of the geometry and a direct proof of our statement.

The configuration space of this fluid is a semidirect product of the diffeomorphisms' group and of the space of all functions on $M: \tilde{\tilde{G}}=\operatorname{Diff}(M)$ $\ltimes C^{\infty}(M)$. Recall that the group structure on $\tilde{\mathrm{G}}$ is defined by the formula:

$$
(\varphi, a) \circ(\psi, b)=\left(\varphi \circ \psi, \varphi_{*} b+a\right)
$$

and the commutator in the Lie algebra $\tilde{\mathscr{G}}$ is

$$
[(v, a),(\omega, b)]=\left([v, \omega], L_{v} b-L_{\omega} a\right),
$$

where $\varphi, \psi \in \operatorname{Diff}(M), \quad a, b \in \mathrm{C}^{\infty}(M), v, \omega \in$ $\operatorname{Vect}(M)$, and the square brackets $[v, \omega]$ denote the ordinary commutator of the vector fields.
The equations of the barotropic fluid are the Hamilton equation on the orbits of the coadjoint representation of this group, where the Hamilton function is

$$
\begin{aligned}
H(v, \rho) & =\int_{M}\left[\frac{1}{2} \rho v^{2}+\varepsilon(\rho)\right] \mu, \\
\dot{\varepsilon}(\rho) & =h(\rho) .
\end{aligned}
$$

The dual space to $\operatorname{Vect}(M)$ can be identified with the tensor product $\Omega^{1}(M) \otimes \Omega^{D}(M)$ (with the natural pairing: the vector field is substituted in the 1-form and the obtained $D$-form is integrated over $M$ ). Hence, elements of $\tilde{\mathscr{G}}^{*}$ are pairs $(\beta, \theta)$, where $\beta \in \Omega^{1}(M) \otimes \Omega^{D}(M)$ and $\theta \in$ $\Omega^{D}(M)$. The coadjoint action of the element $(\varphi, a) \in \operatorname{Diff}(M) \ltimes \mathrm{C}^{\infty}(M)$ is

$\operatorname{Ad}_{(\varphi, a)}^{*}(\beta, \theta)=\left(\varphi_{*} \beta+\mathrm{d} a \otimes \varphi_{*} \theta, \varphi_{*} \theta\right)$.

Note that $u=\beta / \theta$ has the geometric sense of a 1-form.

Proposition 2. The functional $I(\beta, \theta)=\int u \wedge$ $(\mathrm{d} u)^{m}$ in the case $D=2 m+1$ and the functionals $I_{f}(\beta, \theta)=\int f\left((\mathrm{~d} u)^{m} / \theta\right) \theta$ in the case $D=2 m$ (where $u=\beta / \theta \in \Omega^{1}(M)$ ) are invariant under the coadjoint $\tilde{\mathrm{G}}$-action on $\tilde{\mathscr{G}}^{*}$.

Proof.

$$
\begin{aligned}
\operatorname{Ad}_{(\varphi, a)}^{*} u & =\operatorname{Ad}_{(\varphi, a)}^{*}\left(\frac{\beta}{\theta}\right)=\frac{\varphi_{*} \beta+\mathrm{d} a \otimes \varphi_{*} \theta}{\varphi_{*} \theta} \\
& =\varphi_{*}\left(\frac{\beta}{\theta}\right)+\mathrm{d} a=\varphi_{*} u+\mathrm{d} a
\end{aligned}
$$

i.e., the 1-form is transported by the flow modulo a differential of a function; the action on class $[u] \in \Omega^{1} / \mathrm{d} \Omega^{0}$ as well as on $\theta \in \Omega^{D}$ is reduced to the change of variables. Now the proposition (as well as proposition 1 in section 2) follows from the coordinate-free definition of $I$ and $I_{f}$.

The inertia operator $\tilde{A}: \tilde{\mathscr{G}} \rightarrow \tilde{\mathscr{G}}^{*}$, defined by the metrics, admits the following expression: $(v, \rho) \mapsto$ $(u \otimes \theta, \theta)$, where $\theta=\rho \mu$ is the density form and 
1-form $u$ is obtained from $v$ by the "lifting of indices.". Thus, theorem 3 immediately follows from proposition 2 in analogy with section 2 .

\section{The ergodic interpretation of three-dimensional magnetohydrodynamics}

In magnetohydrodynamics we assume that the magnetic field $\boldsymbol{B}$ is "frozen" in the ideal fluid of infinite conductivity filling a manifold $M$. The fluid flow preserves the volume $\mu$ on $M$ induced by the metrics $g$. The velocity field $v$ and the "frozen" magnetic field $B(\operatorname{div} v=\operatorname{div} B=0)$ satisfy the so-called magnetic hydrodynamical equations:

$$
\begin{aligned}
& \dot{\boldsymbol{v}}=-(\boldsymbol{v}, \nabla) \boldsymbol{v}+\operatorname{rot} \boldsymbol{B} \times \boldsymbol{B}+\nabla p, \\
& \dot{\boldsymbol{B}}=[\boldsymbol{v}, \boldsymbol{B}]
\end{aligned}
$$

(the second equation is the definition of the "frozeness" of the field $\boldsymbol{B},[.,$.$] denotes the com-$ mutator of two vector fields).

These equations are connected with the Lie algebra $\mathscr{F}=\mathscr{G} \times \mathscr{G}^{*}$, which is the semidirect product of the Lie algebra $\mathscr{G}$ of all divergence-free vector fields on $M$ with the volume $\mu$ and of its dual space $\mathscr{G}^{*}$. The dual space $\mathscr{F}^{*}$ coincides with $\mathscr{G}^{*} \oplus \mathscr{G}$ as a linear space.

Proposition 3. (Compare with refs. $[10,16]$.)

(i) The equations of the magnetic hydrodynamics (6) are Hamilton equations on the space $\mathscr{F}^{*}$ relative to the standard Poisson bracket.

(ii) The quadratic form $J([u], B)=\int_{M} u(B) \mu$ on $\mathscr{F}^{*}$ is an invariant of the coadjoint representation of the corresponding Lie group $\mathrm{F}=\mathrm{G} \ltimes \mathscr{G}^{*}$ (here $B \in \mathscr{G},[u] \in \Omega^{1} / \mathrm{d} \Omega^{0}=\mathscr{G}^{*}$ and $u \in \Omega^{1}$ is a representative of $[u])$.

\section{Proof.}

(i) By definition the commutator in $\mathscr{G} \ltimes \mathscr{G}^{*}$ is

$$
\begin{aligned}
& {[(v,[\alpha]),(\omega,[\beta])]_{\mathscr{F}}} \\
& \quad=\left([v, \omega]_{\mathscr{S}}, \operatorname{ad}_{v}^{*}[\beta]-\operatorname{ad}_{\omega}^{*}[\alpha]\right)
\end{aligned}
$$

This formula implies the following formula for the coadjoint $\mathscr{T}_{\text {action }}$ on $\mathscr{F}^{*}$ :

$$
\begin{aligned}
& \operatorname{ad}_{(\boldsymbol{v},[\boldsymbol{\alpha}])}^{*}([u], \boldsymbol{B}) \\
& \quad=\left(L_{v}[u]-L_{\boldsymbol{B}}[\alpha],[\boldsymbol{v}, \boldsymbol{B}]_{\mathscr{S}}\right) .
\end{aligned}
$$

An arbitrary metrics on $M$ (see section 2 ) defines an inertia operator $A: \mathscr{G} \rightarrow \mathscr{G}^{*}$, which determines a scalar product on $\mathscr{F}^{*}$. The corresponding quadratic form is

$H_{\text {mag }}([u], \boldsymbol{B})=\frac{1}{2}\left\langle[u], A^{-1}[u]\right\rangle+\frac{1}{2}\langle\boldsymbol{B}, A \boldsymbol{B}\rangle$.

The Hamiltonian equations on $\mathscr{F}^{*}$ with the Hamilton function $H_{\text {mag }}$ is, according to (7),

$[u]^{*}=-L_{v}[u]+L_{B}[b]$,

$\dot{\boldsymbol{B}}=[\boldsymbol{v}, \boldsymbol{B}]_{\mathscr{S}}$,

where $v=A^{-1}[u],[b]=A B$.

One can easily check that if the volume form is defined by the metrics on $M$, then operator $A^{-1}$ maps $L_{\boldsymbol{B}}[b]$ to rot $\boldsymbol{B} \times \boldsymbol{B}$ (see section 3), and, hence, it maps $[u]^{\circ}$ to the vector field

$\dot{v}=-(v, \nabla) v+\operatorname{rot} B \times B+\nabla p$.

This equation together with the relations $\dot{\boldsymbol{B}}=$ $[\boldsymbol{v}, \boldsymbol{B}]_{\mathscr{S}}$ and $\operatorname{div} \boldsymbol{B}=\operatorname{div} \boldsymbol{v}=0$ forms the system of magnetic hydrodynamics equations. Thus, eqs. (8) are their invariant formulation.

(ii) The invariance of the quadratic form $J$ can be verified by the explicit calculation using the following coadjoint action of the group $\mathrm{F}$ :

$$
\begin{aligned}
& \operatorname{Ad}_{(\varphi,[\alpha])}^{*}([u], B) \\
& \quad=\left(\varphi_{*}\left([u]-L_{B}\left(\varphi_{*}^{-1}[\alpha]\right)\right), \varphi_{*} B\right) .
\end{aligned}
$$

Note that the invariance of $J$ with respect to the algebra action implies only the constancy of $J$ on the connected components of the coadjoint orbits. This ends the proof of proposition 3 .

Corollary 5. (See ref. [16].) The value of $\int_{M}(v, B) \mu$ is preserved on the trajectories of the magnetic hydrodynamics equations (8). 
Indeed, these trajectories are the inverse images of the trajectories of (8) on the F-orbits in $\mathscr{F}^{*}$ $\left(v=A^{-1}[u]\right)$, on which (by proposition 3$) \int u(B) \mu$ is preserved.

The main goal of this section is to give an ergodic interpretation of the magnetic hydrodynamics invariants that we have found. At first we give the definition of the average linking number of two divergence-free vector fields [5].

Let $M^{3}$ be a simply connected manifold with volume $\mu$, and $\boldsymbol{\xi}$ and $\eta$ two divergence-free vector fields on $M ; g_{\xi}^{t}$ and $g_{\eta}^{t}$ denote their phase flows. For two points $x, y \in M$ given, we define the "asymptotic linking number" of the trajectories of $g_{\xi}^{t}$ and $g_{\eta}^{t}$ starting in $x$ and $y$, respectively. For this purpose we at first connect any two points on $M$ by a "short" path $\Delta$ (the conditions imposed on the short path will be described below and are satisfied at "almost any" choice of the "short" paths $\Delta$ ).

We select two large numbers $T$ and $S$ and close the segment $g_{\xi}^{t} x(0 \leq t \leq T)$ and $g_{\eta}^{t} y(0 \leq t \leq S)$ of the trajectories issuing from $x$ and $y$ by "short" paths $\Delta\left(g_{\xi}^{T} x, x\right)$ and $\Delta\left(g_{\eta}^{s} y, y\right)$, so that we obtain two closed curves $\Gamma=\Gamma_{T}(x)$ and $\Gamma^{\prime}=\Gamma_{S}(y)$. We assume that these curves are nonintersecting (this is true for almost all pairs of $x$ and $y$, and for almost all $T$ and $S$ ). Then the linking number $N_{T, S}(x, y)$ of $\Gamma$ and $\Gamma^{\prime}$ is defined.

Definition 3. The asymptotic linking number of the pair of the trajectories $g_{\xi}^{t} x$ and $g_{\eta}^{t} y$ is defined as the limit

$\lambda(x, y)=\lim _{T, S \rightarrow \infty} \frac{N_{T, S}(x, y)}{T S}$

( $T$ and $S$ are to vary so that $\Gamma$ and $\Gamma^{\prime}$ do not intersect).

Further we are going to prove that this limit exists almost everywhere and is independent of the system of "short" paths $\Delta$ (as an element of $\left.L_{1}(M \times M)\right)$.
Definition 4. The average linking number $\lambda$ of two divergence-free vector fields is

$\lambda=\int_{M} \int_{M} \lambda\left(x_{1}, x_{2}\right) \mu_{1} \mu_{2}$.

Now we can state the main result, which is a modification of the correspondent statement by Arnol'd [5]:

Theorem 4. The average linking number $\lambda$ of two divergence-free vector fields $\boldsymbol{\xi}$ and $\boldsymbol{\eta}$ on a simply connected three-dimensional manifold $M$. with volume $\mu$ coincides with

$\int_{M} i_{\xi} \mu \wedge \mathrm{d}^{-1}\left(i_{\eta} \mu\right)$

The condition of the vanishing of the divergence for the vector field $\eta$ on $M$ is equivalent to the condition $\mathrm{d} i_{\eta} \mu=0$ or $i_{\eta} \mu=\mathrm{d} \nu$, while the integral $\int i_{\xi} \mu \wedge \nu$ evidently does not depend on the freedom in the choice of $\nu \in \Omega^{1}(M)$.

Corollary 6 . The magnetic hydrodynamics invariant $\int(\boldsymbol{v}, \boldsymbol{B}) \mu$ on a simply connected three-dimensional manifold coincides with the average linking number of the vector fields rot $\boldsymbol{v}$ and $\boldsymbol{B}$. Indeed, applying our theorem to the vector fields $\boldsymbol{\xi}=\boldsymbol{B}$ and $\boldsymbol{\eta}=\operatorname{rot} v$ (and using the relation $[u]=A v, \mathrm{~d} u$ $\left.=i_{\text {rot } v} \mu\right)$, we obtain

$$
\begin{aligned}
\int i_{B} \mu \wedge \mathrm{d}^{-1}\left(i_{\text {rot } v} \mu\right) & =\int i_{B} \mu \wedge \mathrm{d}^{-1}(\mathrm{~d} u) \\
& =\int i_{B} \mu \wedge u=\int u(B) \mu .
\end{aligned}
$$

QED

Note that, in spite of the dependence of $v=$ $A^{-1}[u]$ on the choice of the metrics, the field rot $v$ is defined unambiguously as the kernel field : of $\mathrm{d}[u]$.

The ergodic interpretation of $\int(v, B) \mu$ as of an average linking number of rot $\boldsymbol{v}$ and $B$ is somehow unexpected, since rot $\boldsymbol{v}$ (in contrast to $\boldsymbol{B}$ ) is 
not "frozen" (see eq. (8)). The evolution changes the field $[u]$ (and, hence, $\mathrm{d}[u]$ as well) by some additive summand, which depends on $B$, but it turns out that the average linking number of the kernel field and field $\boldsymbol{B}$ is preserved.

Remark 8. In ref. [5] this result was proved for the coincident $\boldsymbol{\xi}$ and $\eta$ (for this case the integrability of the unbounded functions arising in the proof was checked). This gives an ergodic interpretation for the total helicity integral.

The proof proposed below is a more invariant reformulation of the correspondent argument of ref. [5] and it enables to prove the integrability in the more general case.

Proof. Recall some facts about double bundles and Gauss-type linking forms.

Definition 5. $K \in \Omega^{2}(M \times M)$ is called a linking form on a simply connected manifold $M^{3}$, if for an arbitrary pair of nonintersecting closed curves $\Gamma_{1}$ and $\Gamma_{2}$ we have:

$\int_{\Gamma_{1} \times \Gamma_{2} \subset M \times M} K=N\left(\Gamma_{1}, \Gamma_{2}\right)$

where $N\left(\Gamma_{1}, \Gamma_{2}\right)$ is a linking number of $\Gamma_{1}$ and $\Gamma_{2}$ on $M, \Gamma_{1} \times \Gamma_{2}=\left\{(x, y) \in M \times M \mid x \in \Gamma_{1}, y \in\right.$ $\left.\Gamma_{2}\right\}$. The existence of such a form will be settled later.

Definition 6. Each form $A(x, y) \in \Omega(M \times M)$ determines an operator $\tilde{A}: \Omega(M) \rightarrow \Omega(M)$ such that $\varphi(y) \mapsto \int_{\pi^{-1}(x)} A(x, y) \wedge \varphi(y)$, where $\pi: M \times M$ $\rightarrow M$ is the projection on the first component and the integration is performed over the fibres of this projection.

Proposition 4. An operator $\tilde{K}$ corresponding to the linking form is a Green operator, i.e., an operator inverse to the external derivative: if $\psi=$ $\mathrm{d} \varphi$ and $\varphi \in \Omega^{1}(M)$, then $\varphi=\tilde{K}(\psi)+\mathrm{d} f$ (the summand $\mathrm{d} f$ points that $\varphi$ can be defined by $\psi$ only modulo a full differential).
Proof of proposition 4. Let $\mathrm{D}=\mathrm{d}_{x}+\mathrm{d}_{y}$ be the operator of the external derivative on $\Omega(M \times M)$.

Lemma 4.

$\overline{\mathrm{d}_{x} A}=\mathrm{d} \circ \tilde{A}$.

Indeed,

$\left[\mathrm{d}_{x} A(x, y)\right] \wedge \varphi(y)=\mathrm{d}_{x}[A(x, y) \wedge \varphi(y)]$

and thus,

$$
\begin{aligned}
& \int_{\pi^{-1}(x)}\left[\mathrm{d}_{x} A(x, y)\right] \wedge \varphi(y) \\
& =\mathrm{d}\left(\int_{\pi^{-1}(x)} A(x, y) \wedge \varphi(y)\right) .
\end{aligned}
$$

Lemma 5. If $A$ is a 1 -form in the variable $y$, then $\widetilde{\mathrm{d}} \boldsymbol{A}=\tilde{A} \circ \mathrm{d}$.

Indeed,

$$
\begin{gathered}
\int_{\pi^{-1}(x)}\left[\mathrm{d}_{y} A(x, y)\right] \wedge \varphi(y) \\
=\int_{\pi^{-1}(x)} A(x, y) \wedge \mathrm{d} \varphi(y) .
\end{gathered}
$$

Lemma 6. The external derivative of a linking form is $\mathrm{D} K=\delta+\beta$, where $\delta$ is the $\delta$-form on the diagonal in $M \times M$ and $\beta \in \Omega^{3}(M \times M)$ is a linear combination of closed (and, thus, exact) forms with respect to each of the two arguments.

Proof.

$$
\begin{aligned}
N\left(\Gamma_{1}, \Gamma_{2}\right) & =\int_{\Gamma_{1} \times \Gamma_{2}} K=\int_{\partial^{-1}\left(\Gamma_{1} \times \Gamma_{2}\right)} \mathrm{DK} \\
& =\int_{\left(\partial^{-1} \Gamma_{1}\right) \times \Gamma_{2}} \mathrm{DK} .
\end{aligned}
$$

On the other hand, by the definition of the linking number as the intersecting number of the cycle $\Gamma_{2}$ with a film $\partial^{-1} \Gamma_{1}$ (whose boundary is $\Gamma_{1}$ ) we 
obtain

$N\left(\Gamma_{1}, \Gamma_{2}\right)=\int_{\left(\partial^{-1} \Gamma_{1}\right) \times \Gamma_{2}} \delta$.

Then the statement follows from the fact that

$\int_{\left(\partial^{-1} \Gamma_{1}\right) \times \Gamma_{2}} \beta=0$

Remark 9. In spite of the nonvanishing of the cohomological class of $\delta$ (since the diagonal in $M \times M$ is not a boundary) we can choose $\beta$ satisfying the vanishing condition for the cohomological class of $\delta+\beta$ (the diagonal can be decomposed in the sum of homology groups' generators). Thus, we have proved the existence of a linking form $K$ as the solution of the equation:

$\mathrm{D} K=0 \in H^{3}(M \times M)$.

To finish the proof of proposition 4 we pass in the equation $D K=\delta+\beta$ from the forms to the operators: $\overline{\mathrm{DK}}=\tilde{\delta}+\tilde{\beta}$ or $\widetilde{\mathrm{d}_{x} K}+\widetilde{\mathrm{d}_{y} K}=\tilde{\delta}+\tilde{\beta}$. Since the $\delta$-form corresponds to the identity operator, and the image of the operator $\tilde{\beta}$ belongs to the space of the exact forms by lemmas 4-6 we obtain

$\mathrm{d} \circ \tilde{K}+\tilde{K} \circ \mathrm{d}=i \mathrm{~d}+\mathrm{d} \circ \tilde{\gamma}$

Applying these operators to the form $\varphi$ we get

$\mathrm{d} \circ \tilde{K}(\varphi)+\tilde{K}(\mathrm{~d} \varphi)=\varphi+\mathrm{d} \circ \tilde{\gamma}(\varphi)$.

Since $\psi=d \varphi$, the final formula is

$\varphi=\tilde{K}(\psi)+\mathrm{d} f$

QED

Lemma 7. There is the linking form $K(x, y)$ having a pole of degree 2 on the diagonal of $M \times M$, i.e. its coefficients grow as $c /|x-y|^{2}$ (we use an arbitrary metrics on $M \times M$ induced by a metrics on $M$ ).
Proof. In fact, the linking number of $\Gamma_{1}$ and $\Gamma_{2}$ coincides with the linking number of $\Gamma_{1} \times \Gamma_{2}$ and of the diagonal in $M \times M$. We identify a neighbourhood of the diagonal in $M \times M$ and a neighbourhood of zero-section in normal bundle of the diagonal (i.e. in $T_{1} M$ ) by the exponential map. Then in every fibre (that is a neighbourhood of $0 \in \mathbb{R}^{3}$ ) we consider the standard linking form with the point 0 . This linking form coincides with substitution of vector field $\nabla(-1 / r)=(\nabla r) / r^{3}$ (with a pole in 0 of degree 2) to the standard volume form in a fibre. At least, let this form be vanished on vectors parallel to $M \subset T_{\perp} M$. So, the corresponding linking form in $M \times M$ has a pole of degree 2 on the diagonal.

QED

Corollary 7. The linking form $K$ is integrable, i.e. the value of $K$ evaluated at any two smooth vector fields is an integrable function on $M \times M(K \in$ $\left.L_{1}(M \times M)\right)$.

Indeed, the codimension of the diagonal in $M$ $\times M$ equals 3 and the order of growth of $K$ near the diagonal equals 2 .

Note that all the previous arguments on linking forms hold (with some evident modifications) for the manifolds of any dimension. The further considerations are essentially three-dimensional. Thus, we have two divergence-free fields $\xi$ and $\eta$ on $M$, equipped with the volume form $\mu$.

Definition 7. (See ref. [5].) A system of short paths joining the points $x$ and $y \in M$ is a system of paths depending in a measurable way on $x$ and $y$, such that the integrals of the linking form $K$ for every pair of nonintersecting paths of the system, and also for any nonintersecting pair (path of the system, unitary time segment of phase curve of fields $\boldsymbol{\xi}$ or $\boldsymbol{\eta}$ ) are bounded independently of the paths by a constant $C$.

Such systems exist since the integral of $K$ for the pair of paths remains bounded when these paths approach "transversally."

Let $g_{\xi}^{t} x$ and $g_{\eta}^{t} y$ be the trajectories of the fields $\xi$ and $\eta$ starting from $x$ and $y$ for the time 
intervals $0 \leq t \leq T$ and $0 \leq t \leq S, \Delta_{x}$ and $\Delta_{y}$ be the corresponding short paths closing these trajectories. Now

$$
\begin{aligned}
\lambda(x, y) & =\lim _{T, S \rightarrow \infty} \frac{1}{T S} \int_{\left(g_{\xi}^{t} x+\Delta_{x}\right) \times\left(g_{\eta}^{t} y+\Delta_{y}\right)} K \\
& =\lim _{T, S \rightarrow \infty} \frac{1}{T S} \int_{g_{\xi}^{t} x \times g_{\eta}^{t} y} K .
\end{aligned}
$$

This equality of limits follows from the boundness of the integral over the short paths. Then

$$
\begin{aligned}
& \iint_{M \times M} \lambda(x, y) \mu_{x} \mu_{y} \\
& \quad=\int_{M} \mu_{x} \int_{M} \mu_{y} \lim _{T, S \rightarrow \infty} \frac{1}{T S} \int_{g_{\xi}^{t} x \times g_{\eta}^{2} y} K \\
& \quad=\int_{M} \mu_{x} \int_{M} \mu_{y}\left(\lim _{T, S \rightarrow \infty} \frac{1}{T S} \int_{0}^{T} \int_{0}^{S}\left(i_{\xi} i_{\eta} K\right) \mathrm{d} t \mathrm{~d} s\right)
\end{aligned}
$$

where $\int_{0}^{T}$ and $\int_{0}^{S}$ denote the integrals of the function $i_{\xi} i_{\eta} K$ along the trajectories $g_{\xi}^{t} x$ and $g_{\eta}^{t} y$. By the Birkhoff ergodic theorem we can pass from the time averages to the space average:

$$
\iint_{M \times M} \lambda(x, y) \mu_{x} \mu_{y}=\int_{M} \mu_{x} \int_{M} \mu_{y}\left(i_{\xi} i_{\eta} K\right)
$$

(recall that $K \in L_{1}(M \times M)$ ). Recalling the definition of the average linking number $\lambda$ as the average value of $\lambda(x, y)$ over $M \times M$ and shifting the operators of substitution $i_{\xi}$ and $i_{\eta}$ to the forms $\mu_{x}$ and $\mu_{y}$ we obtain the following:

$$
\begin{aligned}
\lambda & =\iint_{M \times M} \lambda(x, y) \mu_{x} \mu_{y}=\int_{M} \mu_{x} \int_{M} \mu_{y}\left(i_{\xi} i_{\eta} K\right) \\
& =\int_{M} i_{\xi} \mu_{x} \wedge\left(\int_{M} i_{\eta} \mu_{y} \wedge K\right) \\
& =\int_{M} i_{\xi} \mu \wedge \tilde{K}\left(i_{\eta} \mu\right) .
\end{aligned}
$$

By proposition $4 \tilde{K}\left(i_{\eta} \mu\right)=\mathrm{d}^{-1}\left(i_{\eta} \mu\right)$ modulo an exact form, i.e. $\lambda=\int i_{\xi} \mu \wedge \mathrm{d}^{-1}\left(i_{\eta} \mu\right)$. The proof of theorem 4 is completed.
It would be very interesting to find the multidimensional generalization of this ergodic interpretation. Probably, this interpretation is connected with the symplectic properties of the space of the trajectories of a divergence-free vector field and our integral invariants would be in some sense the "asymptotic" Maslov indices.

\section{Acknowledgements}

We are profoundly grateful to V.I. Arnol'd for the proposal of the problems and for many fruitful discussions of the ideas treated here. The results of section 2 are obtained jointly with V.Yu. Ovsienko [14]. We are grateful to him and also to I.S. Zaharevich and V.V. Fock for helpful discussions.

\section{References}

[1] V.I. Arnol'd, Sur la géométrie différentielle des groupes de Lie de dimension infinite et ses applications à l'hydrodynamique des fluides parfaits, Ann. Inst. Fourier 16 (1966) 319.

[2] V.I. Arnol'd, Hamilton character of the Euler equations of solid body and ideal fluid, Usp. Mat. Nauk 24 (3) (1969) 225 (in Russian).

[3] V.I. Amol'd, On one-dimensional cohomology of Lie algebra of divergence-free vector fields and on rotation number of dynamical systems, Funct. Anal. Appl. 3 (1969) 4.

[4] V.I. Arnol'd, Mathematical Methods of Classical Mechanics (Springer, Berlin, 1978).

[5] V.I. Arnol'd, The asymptotic Hopf invariant and its applications (1974) (in Russian), English transl. Sel. Math. Sov. 5 (1986) 327.

[6] A.A. Dezin, Invariant forms and some structure properties of the Euler equations of hydrodynamics, Z. Anal. Anwend. 2 (1983) 401 (in Russian).

[7] J. Marsden and A. Weinstein, Coadjoint orbits, vortices, and Clebsch variables for incompressible fluids, Physica D 7 (1983) 305.

[8] B.A. Dubrovin, S.P. Novikov and A.T. Fomenko, Modern geometry (Nauka, Moscow, 1979) (in Russian).

[9] R.P. Feynman, Statistical Mechanics (Benjamin, New York, 1972).

[10] D.D. Holm and B.A. Kupershmidt, Poisson brackets and Clebsch representations for magnetohydrodynamics, multifluid plasmas, and elasticity, Physica D 6 (1983) 347.

[11] D.D. Holm and B.A. Kupershmidt, Poisson structures of superconductors, Phys. Lett. A 93 (1983) 177. 
[12] D.D. Holm, J.E. Marsden, T. Ratiu and A. Wienstein, Nonlinear stability conditions and a priori estimates for barotropic hydrodynamics, Phys. Lett. A 98 (1983) 15.

[13] P.J. Olver, A nonlinear Hamiltonian structure for the Euler equations, J. Math. Anal. Appl. 89 (1982) 233.

[14] V.Yu. Ovsienko, B.A. Khesin and Yu.V. Chekanov, Integrals of the Euler equations of multidimensional hydrodynamics and superconductivity, Zapiski LOMI 172 (1989)
105 (in Russian), Engl. transl. in J. Sov. Math., to appear. [15] D. Serre, Invariants et dégénérescence symplectique de l'équation d'Euler des fluides parfaits incompressibles, C. R. Acad. Sci. (Paris) Ser. A. 298 (1984) 349.

[16] S.V. Vishik and F.V. Dolzanskii, Analogs of the EulerPoisson equations and magnetohydrodynamics equations connected with Lie groups, Dokl. Akad. Nauk USSR 238 (1978) 1032. 